\title{
A MAX-MIN DESIGN OF TRANSMIT SEQUENCE AND RECEIVE FILTER
}

\author{
Mohammad Mahdi Naghsh ${ }^{*+}$, Mojtaba Soltamalian ${ }^{++}$, Petre Stoica $^{++}$, Mahmoud Modarres-Hashemi $^{+}$, \\ Antonio De Maio ${ }^{\dagger}$, and Augusto Aubry ${ }^{\dagger \dagger}$ \\ ${ }^{+}$ECE Dept., Isfahan University of Technology, Isfahan, Iran \\ ${ }^{++}$Dept. of Information Technology, Uppsala University, Uppsala, Sweden \\ †DIETI, Università degli Studi di Napoli “Federico II”, Napoli, Italy \\ ${ }^{\dagger} \mathrm{CNR}$, IREA, Napoli, Italy
}

\begin{abstract}
In this paper, we study the joint design of Doppler robust transmit sequence and receive filter to improve the performance of an active sensing system dealing with signaldependent interference. The signal-to-interference-plus-noise ratio (SINR) of the filter output is considered as the performance measure of the system. The design problem is cast as a max-min optimization problem to robustify the system SINR with respect to the unknown Doppler shifts of the targets. To tackle the design problem, we devise a novel method to obtain optimized pairs of transmit sequence and receive filter sharing the desired robustness property.

Keywords: Doppler shift, max-min, receive filter, robust design, transmit sequence
\end{abstract}

\section{INTRODUCTION}

The performance of an active sensing system can be significantly improved by judiciously designing its transmit sequence and receive filter. Such a design usually encompasses several challenges including the fact that Doppler shifts of moving targets are often unknown at the transmit side, the existence of signal-dependent interference (clutter) as well as signal-independent interference at the receive side, and practical constraints such as similarity to a given code.

Joint design of the transmit sequence and the receive filter has been considered in a large number of studies during the last decades. Most of the works have been concerned with either stationary targets or targets with known Doppler shifts (see e.g. [1-5]). In [6], considering a stationary target, a frequency domain approach has been employed to obtain an optimal receive filter and corresponding optimal energy spectral density of the transmit signal; then a synthesis procedure has been used to approximately provide the time domain signal. The work of [7] considers a related problem to that of [6] under a peak-to-average power ratio (PAR) constraint. The

This work was supported in part by the European Research Council (ERC) under Grant \#228044 and the Swedish Research Council. *Please address all the correspondence to Mohammad Mahdi Naghsh, Phone: +983113912450; Fax: +983113912451; Email: mm_naghsh@ec.iut.ac.ir reference [8] deals with joint design of transmit sequence and receive filter under a similarity constraint in cases where the Doppler shift of the target is known (see also [9]). In [10], the unknown target Doppler shift has been dealt with via employing an average approach.

Several researches consider signal-independent clutter scenarios (see e.g. [11]). The unknown Doppler shift of the target has been taken into account in [11].

In this paper, we devise a novel method for Doppler robust joint design of transmit sequence and receive filter of a radar system in the presence of (signal-dependent) clutter. We consider the SINR at the output of the receive filter as the performance measure of the system. Besides an energy constraint, a similarity constraint is imposed on the transmit sequence to control certain characteristics of the transmit waveform. The design problem is cast as a max-min optimization to robustify the system performance. We devise a cyclic maximization to tackle a relaxed version of the design problem as well as a synthesis stage to obtain an optimized solution to the problem.

The rest of this paper is organized as follows. The problem formulation is presented in Section 2. Section 3 contains the steps for the derivation of the proposed method.Numerical results are provided in Section 4. Finally, conclusions are drawn in Section 5.

\section{PROBLEM FORMULATION}

We consider a radar system with transmit sequence $\mathbf{x} \in \mathbb{C}^{N}$ and receive filter $\mathbf{w} \in \mathbb{C}^{N}$. The discrete-time received signal backscattered from a moving target corresponding to the range-azimuth cell under the test can be modeled as (see, e.g. [8]):

$$
\mathbf{r}=\alpha_{T} \mathbf{x} \odot \mathbf{p}(\nu)+\mathbf{c}+\mathbf{n},
$$

where $\alpha_{T}$ is a complex parameter associated with backscattering effects of the target as well as propagation effects, $\mathbf{p}(\nu)=\left[1, e^{j \nu}, \ldots, e^{j(N-1) \nu}\right]^{T}$ with $\nu$ being the normalized target Doppler shift, $\mathbf{c}$ is the $N$-dimensional column vector containing clutter samples, and $\mathbf{n}$ is the $N$-dimensional column vector of interference samples. The SINR at the output 
of the receive filter can be formulated as

$$
\operatorname{SINR}(\nu)=\frac{\left|\alpha_{T}\right|^{2}\left|\mathbf{w}^{H}(\mathbf{x} \odot \mathbf{p}(\nu))\right|^{2}}{\mathbf{w}^{H} \mathbf{\Sigma}_{\mathbf{c}}(\mathbf{x}) \mathbf{w}+\mathbf{w}^{H} \mathbf{M} \mathbf{w}}
$$

where $\mathbf{M} \triangleq \mathrm{E}\left\{\mathbf{n} \mathbf{n}^{H}\right\}$ and $\boldsymbol{\Sigma}_{\mathbf{c}}(\mathbf{x})$ is the covariance matrix of c.

We consider the SINR in (2) as the performance measure of the system and aim to find a robust design of the transmit sequence and the receive filter with respect to the unknown Doppler shift of the target. In addition to an energy constraint, a similarity constraint is imposed on the transmit sequence and hence the design problem can be cast as:

$$
\mathcal{P}\left\{\begin{array}{cl}
\max _{\mathbf{x}, \mathbf{w}} \min _{\nu \in \Omega} & \frac{\left|\mathbf{w}^{H}(\mathbf{x} \odot \mathbf{p}(\nu))\right|^{2}}{\mathbf{w}^{H} \mathbf{\Sigma}_{\mathbf{c}}(\mathbf{x}) \mathbf{w}+\mathbf{w}^{H} \mathbf{M} \mathbf{w}} \\
\text { subject to } & \|\mathbf{x}\|^{2}=e \\
& \left\|\mathbf{x}-\mathbf{x}_{0}\right\|^{2} \leq \delta
\end{array}\right.
$$

where $\mathbf{x}_{0}$ is the given code associated with the similarity constraint, $\Omega=\left[\nu_{l}, \nu_{u}\right] \subseteq[-\pi, \pi]$ denotes a given interval of the target Doppler shift $\nu$ and $e$ denotes the maximum available transmit energy. Let $\mathbf{X}=\mathbf{x x}^{H}$ and $\mathbf{W}=\mathbf{w} \mathbf{w}^{H}$. Using standard properties of the Hadamard product and Lemma 3.1 in [8], $\operatorname{SINR}(\nu)$ can be alternatively expressed as follows (the proof is omitted due to the lack of space):

$$
\begin{aligned}
\operatorname{SINR}(\nu) & =\frac{\left|\alpha_{T}\right|^{2} \mathbf{p}(\nu)^{H}\left(\mathbf{W} \odot \mathbf{X}^{*}\right) \mathbf{p}(\nu)}{\operatorname{tr}\left\{\left(\mathbf{\Sigma}_{\mathbf{c}}(\mathbf{X})+\mathbf{M}\right) \mathbf{W}\right\}} \\
& =\frac{\left|\alpha_{T}\right|^{2} \mathbf{p}(\nu)^{H}\left(\mathbf{W} \odot \mathbf{X}^{*}\right) \mathbf{p}(\nu)}{\operatorname{tr}\left\{\left(\Theta_{\mathbf{c}}(\mathbf{W})+\left(\frac{\beta}{e}\right) \mathbf{I}\right) \mathbf{X}\right\}}
\end{aligned}
$$

where $\beta=\operatorname{tr}\{\mathbf{M W}\}$, and

$$
\begin{gathered}
\boldsymbol{\Sigma}_{\mathbf{c}}(\mathbf{X})=\sum_{k=0}^{N_{c}-1} \sum_{i=0}^{L-1} \sigma_{(k, i)}^{2} \mathbf{J}_{k}\left(\mathbf{X} \odot \boldsymbol{\Phi}_{\epsilon_{(k, i)}}^{\bar{\nu}_{d_{(k, i)}}}\right) \mathbf{J}_{k}^{T}, \\
\boldsymbol{\Theta}_{\mathbf{c}}(\mathbf{W})=\sum_{k=0}^{N_{c}-1} \sum_{i=0}^{L-1} \sigma_{(k, i)}^{2}\left(\mathbf{J}_{k}^{T} \mathbf{W} \mathbf{J}_{k}\right) \odot\left(\boldsymbol{\Phi}_{\epsilon_{(k, i)}}^{\bar{\nu}_{d_{(k, i)}}}\right)^{*}
\end{gathered}
$$

with $N_{c} \leq N$ being the number of range rings that interfere with the range-azimuth bin of interest $(0,0), L$ is the number of discrete azimuth sectors, $\sigma_{(k, i)}^{2}$ is the mean interfering power associated with the clutter patch located at the $(k, i)^{t h}$ range-azimuth bin whose Doppler shift is supposed to be uniformly distributed in the interval $\Omega_{c}=\left(\bar{\nu}_{d_{(k, i)}}-\frac{\epsilon_{(k, i)}}{2}, \bar{\nu}_{d_{(k, i)}}+\frac{\epsilon_{(k, i)}}{2}\right)$. Herein we have

$\boldsymbol{\Phi}_{\epsilon_{(k, i)}}^{\bar{\nu}_{d_{(k, i)}}}(l, m)=\left\{\begin{array}{cl}1, & l=m \\ e^{\left(j(l-m) \bar{\nu}_{d_{(k, i)}}\right) \frac{\sin \left[0.5(l-m) \epsilon_{(k, i)}\right]}{\left[0.5(l-m) \epsilon_{(k, i)}\right]},} & l \neq m\end{array}\right.$

and $\mathbf{J}_{k}$ denotes the aperiodic shift matrix [8] for $0 \leq k \leq$ $N_{c}-1$.

Using (4), we cast the design problem w.r.t (X, W) and relax the rank-one constraints on these matrices. Then, we consider the following problem:

$$
\mathcal{P}_{1} \begin{cases}\max _{\mathbf{X}, \mathbf{W}} \min _{\nu \in \Omega} & \frac{\mathbf{p}(\nu)^{H}\left(\mathbf{W} \odot \mathbf{X}^{*}\right) \mathbf{p}(\nu)}{\operatorname{tr}\left\{\left(\mathbf{\Sigma}_{\mathbf{c}}(\mathbf{X})+\mathbf{M}\right) \mathbf{W}\right\}} \\ \text { subject to } & \operatorname{tr}\{\mathbf{X}\}=e \\ & \operatorname{tr}\left\{\mathbf{X} \mathbf{X}_{0}\right\} \geq \epsilon_{\delta} \\ & \mathbf{X} \succeq \mathbf{0}, \mathbf{W} \succeq \mathbf{0}\end{cases}
$$

where $\mathbf{X}_{0}=\mathbf{x}_{0} \mathbf{x}_{0}^{H}$ and $\epsilon_{\delta}=((2 e-\delta) / 2)^{2}$. Note that optimized solutions to the design problem $\mathcal{P}$ can be obtained from optimal solutions to the above problem, see below.

\section{THE PROPOSED METHOD TO TACKLE THE DESIGN PROBLEM $\mathcal{P}$}

In this section, we devise a novel method to tackle the nonconvex optimization problem $\mathcal{P}$. The method includes solving the relaxed problem $\mathcal{P}_{1}$ via a cyclic maximization approach followed by a synthesis stage.

- Optimal X for fixed W: Let $\widetilde{t} \in \mathbb{R}$ denote a slack variable. For fixed $\mathbf{W}$, the optimization in (6) is equivalent to the following maximization problem:

$$
\mathcal{P}_{X} \begin{cases}\max _{\mathbf{X}, \widetilde{t}} & \frac{\operatorname{tr}\left\{\left(\boldsymbol{\Theta}_{\mathbf{c}}(\mathbf{W})+\left(\frac{\beta}{e}\right) \mathbf{I}\right) \mathbf{X}\right\}}{\text { subject to }} \\ & \mathbf{p}(\nu)^{H}\left(\mathbf{W} \odot \mathbf{X}^{*}\right) \mathbf{p}(\nu) \geq \widetilde{t}, \forall \nu \in \Omega \\ & \operatorname{tr}\{\mathbf{X}\}=e \\ & \operatorname{tr}\left\{\mathbf{X} \mathbf{X}_{0}\right\} \geq \epsilon_{\delta} \\ & \mathbf{X} \succeq \mathbf{0} .\end{cases}
$$

Note that problem $\mathcal{P}_{X}$ is a linear-fractional maximization problem with infinitely many constraints (see the first constraint). Inspired by Charnes-Cooper transform [13], we let $\mathbf{Y}=s \mathbf{X}, t=s \tilde{t}$ for an auxiliary variable $s \geq 0$; it can be shown that the following problem is equivalent to $\mathcal{P}_{X}$ :

$$
\mathcal{P}_{X}^{\prime} \begin{cases}\max _{\mathbf{Y}, t, s} & t \\ \text { subject to } & \operatorname{tr}\left\{\left(\boldsymbol{\Theta}_{\mathbf{c}}(\mathbf{W})+\left(\frac{\beta}{e}\right) \mathbf{I}\right) \mathbf{Y}\right\}=1 \\ & \mathbf{p}(\nu)^{H}\left(\mathbf{W} \odot \mathbf{Y}^{*}\right) \mathbf{p}(\nu) \geq t, \forall \nu \in \Omega \\ & \operatorname{tr}\{\mathbf{Y}\}=e s \\ & \operatorname{tr}\left\{\mathbf{Y} \mathbf{X}_{0}\right\} \geq \epsilon_{\delta} s \\ & \mathbf{Y} \succeq \mathbf{0}, s \geq 0 .\end{cases}
$$

To deal with the constraint set, we note that the constraint $\mathbf{p}(\nu)^{H}\left(\mathbf{W} \odot \mathbf{Y}^{*}\right) \mathbf{p}(\nu) \geq t, \forall \nu \in \Omega$ implies the nonnegativity of a trigonometric polynomial of $\nu$ over the interval $\Omega$. More specifically, let $z_{k} \triangleq \sum_{i=1}^{N-k} Z_{i+k, i}$ for $0 \leq k \leq N-1$ with $\mathbf{Z}=\mathbf{W} \odot \mathbf{Y}^{*}$. It is straightforward to verify that for any $\nu \in \Omega$, the aforementioned constraint is equivalent to $h(\nu) \triangleq z_{0}-t+2 \Re \sum_{k=1}^{N-1} z_{k} e^{-j k \nu} \geq 0$.

We employ a semidefinite representation of the above constraint using Theorem 3.4 in [14] (see also [15]). In particular, let $\mathbf{z}=\left[z_{0}, z_{1}, \ldots, z_{N-1}\right]^{T}$; there should exist an $N \times N$ Hermitian matrix $\mathbf{Z}_{1} \succeq \mathbf{0}$ and an $(N-1) \times(N-1)$ Hermitian matrix $\mathbf{Z}_{2} \succeq \mathbf{0}$ such that

$$
\mathbf{z}=t \mathbf{e}_{1}+\mathbf{F}_{1}^{H}\left(\operatorname{diag}\left(\mathbf{F}_{1} \mathbf{Z}_{1} \mathbf{F}_{1}^{H}\right)+\mathbf{q} \odot \operatorname{diag}\left(\mathbf{F}_{2} \mathbf{Z}_{2} \mathbf{F}_{2}^{H}\right)\right)
$$

where $\mathbf{q}=\left[q_{0}, q_{1}, \ldots, q_{n-1}\right]^{T}$ with $q_{k}=\cos \left(2 \pi k / n-\left(\nu_{l}+\right.\right.$ $\left.\left.\nu_{u}\right) / 2\right)-\cos \left(\left(\nu_{u}+\nu_{l}\right) / 2\right), \mathbf{F}_{1}=\left[\mathbf{f}_{0}, \ldots, \mathbf{f}_{N-1}\right]$ and $\mathbf{F}_{2}=$ $\left[\mathbf{f}_{0}, \ldots, \mathbf{f}_{N-2}\right]$ in which $\mathbf{f}_{k}=\left[1, e^{-j k \theta}, \ldots, e^{-j(n-1) k \theta}\right]^{T}$ 
with $\theta=2 \pi / n$, and $n=2 N-1$. Consequently, $\mathcal{P}_{X}^{\prime}$ is equivalent to the following semidefinite program (SDP):

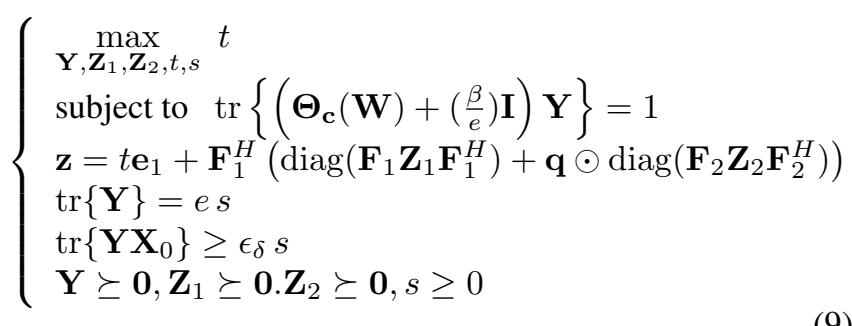

Let $\left(\mathbf{Y}, \mathbf{Z}_{1}, \mathbf{Z}_{2}, t, s\right)$ denote an optimal solution to the above SDP. The corresponding optimal $\mathbf{X}$ (i.e., an optimal solution to $\mathcal{P}_{X}$ ) for fixed $\mathbf{W}$ is given by $\mathbf{Y} / s$.

- Optimal W for fixed X: Using (5) and techniques similar to those for fixed $\mathbf{W}$, we obtain the following SDP for fixed $\mathbf{X}$ :

$$
\left\{\begin{array}{l}
\max _{\mathbf{W}, \mathbf{Z}_{1}^{\prime}, \mathbf{Z}_{2}^{\prime}, \breve{t}} \breve{t} \\
\text { subject to } \operatorname{tr}\left\{\left(\boldsymbol{\Sigma}_{\mathbf{c}}(\mathbf{X})+\mathbf{M}\right) \mathbf{W}\right\}=1 \\
\mathbf{z}^{\prime}=\breve{t} \mathbf{e}_{1}+\mathbf{F}_{1}^{H}\left(\operatorname{diag}\left(\mathbf{F}_{1} \mathbf{Z}_{1}^{\prime} \mathbf{F}_{1}^{H}\right)+\mathbf{q} \odot \operatorname{diag}\left(\mathbf{F}_{2} \mathbf{Z}_{2}^{\prime} \mathbf{F}_{2}^{H}\right)\right) \\
\mathbf{W} \succeq \mathbf{0}, \mathbf{Z}_{1}^{\prime} \succeq \mathbf{0}, \mathbf{Z}_{2}^{\prime} \succeq \mathbf{0}
\end{array}\right.
$$

where $\mathbf{z}^{\prime}$ includes $z_{k}^{\prime}=\sum_{i=1}^{N-k} Z_{i+k, i}^{\prime}$ for $\mathbf{Z}^{\prime}=\mathbf{W} \odot \mathbf{X}^{*}$.

- The synthesis stage: A judicious synthesis of the optimized transmit sequence $\mathbf{x}_{\star}$ and receive filter $\mathbf{w}_{\star}$ from the obtained $\left(\mathbf{W}_{\star}, \mathbf{X}_{\star}\right)$ (via the above cyclic algorithm) is required to maintain the Doppler robustness. If $\mathbf{W}_{\star}$ is rankone, $\mathbf{w}_{\star}$ is available via considering $\mathbf{W}_{\star}=\mathbf{w}_{\star} \mathbf{w}_{\star}^{H}$; whereas if $\mathbf{X}_{\star}=\mathbf{x} \mathbf{x}^{H}$, for $\mathbf{x}_{\star}$ we have $\mathbf{x}_{\star}=\mathbf{x} e^{j \arg \left(\mathbf{x}^{H} \mathbf{x}_{0}\right)}$ [16]. In cases where the rank of either $\mathbf{W}_{\star}$ or $\mathbf{X}_{\star}$ is larger than one, the synthesis of $\mathbf{w}_{\star}$ or $\mathbf{x}_{\star}$ is more complicated. To tackle the synthesis problem, we exploit the rank-one decomposition method [17, Theorem 2.3]; precisely, a rank-one matrix $\mathbf{x} \mathbf{x}^{H}$ can be constructed such that

$$
\mathbf{x}^{H} \mathbf{A}_{i} \mathbf{x}=\operatorname{tr}\left\{\mathbf{X} \mathbf{A}_{i}\right\}, \quad i=1,2,3,4 .
$$

where $\mathbf{X}$ denotes a given Hermitian positive semidefinite matrix and $\left\{\mathbf{A}_{1}, \mathbf{A}_{2}, \mathbf{A}_{3}, \mathbf{A}_{4}\right\}$ are Hermitian matrices (satisfying some mild conditions).

Let $\left(\mathbf{W}_{\star}, \mathbf{X}_{\star}\right)$ denote an optimal solution to $\mathcal{P}_{1}$, and let

$$
\nu_{\star}=\operatorname{argmin}_{\nu \in \Omega} \mathbf{p}(\nu)^{H}\left(\mathbf{W}_{\star} \odot \mathbf{X}_{\star}^{*}\right) \mathbf{p}(\nu) .
$$

Considering the above Theorem and the problem $\mathcal{P}_{X}$, a suitable rank-one matrix $\mathbf{x}_{\star} \mathbf{x}_{\star}^{H}$ can be found such that

$$
\left\{\begin{array}{l}
\left.\operatorname{tr}\{\underbrace{(\underbrace{}_{c}\left(\mathbf{W}_{\star}\right)+(\beta / e) \mathbf{I}}_{\mathbf{R}_{1}}) \mathbf{X}_{\star}\right\}=\mathbf{x}_{\star}^{H} \mathbf{R}_{1} \mathbf{X}_{\star} \\
\left.\operatorname{tr}\{\underbrace{\left(\mathbf { W } _ { \star } \odot \left(\left(\mathbf{p}\left(\nu_{\star}\right) \mathbf{p}\left(\nu_{\star}\right)^{H}\right)^{*}\right.\right.}_{\mathbf{R}_{2}}) \mathbf{X}_{\star}\right\}=\mathbf{x}_{\star}^{H} \mathbf{R}_{2} \mathbf{X}_{\star} \\
\operatorname{tr}\left\{\mathbf{X}_{0} \mathbf{X}_{\star}\right\}=\mathbf{x}_{\star}^{H} \mathbf{X}_{0} \mathbf{X}_{\star} \\
\operatorname{tr}\left\{\mathbf{X}_{\star}\right\}=\mathbf{x}_{\star}^{H} \mathbf{X}_{\star}
\end{array}\right.
$$

We denote the vector obtained via above Theorem by $\mathbf{x}_{\star}=$ $\mathcal{D}\left(\mathbf{X}_{\star}, \mathbf{R}_{1}, \mathbf{R}_{2}, \mathbf{X}_{0}, \mathbf{I}\right)$. Similarly, an optimized receive filter $\mathbf{w}_{\star}$ is available via using $\mathbf{w}_{\star}=\mathcal{D}\left(\mathbf{W}_{\star}, \mathbf{Q}_{1}, \mathbf{Q}_{2}, \mathbf{Q}_{3}, \mathbf{Q}_{4}\right)$ where

$$
\left\{\begin{array}{l}
\mathbf{Q}_{1} \triangleq \mathbf{\Sigma}_{c}\left(\mathbf{X}_{\star}\right)+\mathbf{M} \\
\mathbf{Q}_{2} \triangleq \mathbf{X}_{\star} \odot\left(\mathbf{p}\left(\nu_{\star}\right) \mathbf{p}\left(\nu_{\star}\right)^{H}\right) \\
\mathbf{Q}_{3} \triangleq \mathbf{X}_{\star} \odot\left(\mathbf{p}\left(\nu^{\prime}\right) \mathbf{p}\left(\nu^{\prime}\right)^{H}\right) \\
\mathbf{Q}_{4} \triangleq \mathbf{X}_{\star} \odot\left(\mathbf{p}\left(\nu^{\prime \prime}\right) \mathbf{p}\left(\nu^{\prime \prime}\right)^{H}\right)
\end{array}\right.
$$

Herein $\nu^{\prime}$ and $\nu^{\prime \prime}$ are two arbitrary Doppler shifts in $\Omega$. Employing these points leads to considering the behavior of the SINR associated with the optimal solution w.r.t $\nu$ in three points and so a better synthesis (as compared to considering just $\left.\nu_{\star}\right)$.

Table 1 summarizes the steps of the proposed method for max-min design of transmit sequence and receive filter. Steps 1 and 2 are related to the devised cyclic algorithm and are handled via solving the two SDPs stated in (9) and (10). Steps 4 and 5 aim to synthesize optimized pair of transmit code and receive filter using Theorem 2.3 in [17]. Herein we remark on the fact that the ranks of $\mathbf{W}_{\star}$ and $\mathbf{X}_{\star}$ depend on the employed starting point in addition to the parameters of the design problem. Therefore, for a fixed design problem, it is possible to try several random initiations and choose the best result (considering the ranks of the solutions).

Table 1. Proposed method for max-min design of transmit sequence and receive filter

\footnotetext{
Step 0: Initialize $\mathbf{X}$ with $\mathbf{x x}{ }^{H}$ where $\mathbf{x}$ is a random vector in $\mathbb{C}^{N}$.

Step 1: Solve SDP in (10) to obtain $\mathbf{W}$.

Step 2: Solve SDP in (9) to obtain $\mathbf{X}$.

Step 3: Repeat steps 1 and 2 until a pre-defined stop criterion is satisfied, e.g. $\left\|\mathbf{X}^{(\kappa+1)}-\mathbf{X}^{(\kappa)}\right\|_{F} \leq \mu$ for a given $\mu>0$ where $\kappa$ denotes the iteration number

Step 4 (Receive filter synthesis): If $\mathbf{W}_{\star}$ is rank-one, perform an eigendecomposition $\mathbf{W}_{\star}=\mathbf{w}_{\star} \mathbf{w}_{\star}^{H}$ to obtain $\mathbf{w}_{\star}$. Otherwise, define $\mathbf{w}_{\star}=$ $\mathcal{D}\left(\mathbf{W}_{\star}, \mathbf{Q}_{1}, \mathbf{Q}_{2}, \mathbf{Q}_{3}, \mathbf{Q}_{4}\right)$.

Step 5 (Transmit sequence synthesis): If $\mathbf{X}_{\star}$ is rank-one, perform an eigen-decomposition $\mathbf{X}_{\star}=\mathbf{x} \mathbf{x}^{H}$ to obtain $\mathbf{x}_{\star}=\mathbf{x} e^{j \arg \left(\mathbf{x}^{H} \mathbf{x}_{0}\right)}$. Otherwise, define $\mathbf{x}_{\star}=\mathcal{D}\left(\mathbf{X}_{\star}, \mathbf{R}_{1}, \mathbf{R}_{2}, \mathbf{X}_{0}, \mathbf{I}\right)$.
}

\section{NUMERICAL EXAMPLES}

In this section we provide several numerical examples to examine the effectiveness of the proposed method. Throughout the simulations, we consider a sequence length $N=20$, number of interfering range rings $N_{c}=2$, and number azimuth sectors $L=100$. The interfering signals backscattered from various azimuth sectors are weighted according to the azimuth beam-pattern characteristic of a typical linear array (see [8] for details). A uniformly distributed clutter is assumed with $\sigma_{(k, i)}^{2}=\sigma^{2}=100$ for all $(k, i)$. As to the target, we set $\alpha_{T}=1$. Concerning the covariance matrix 


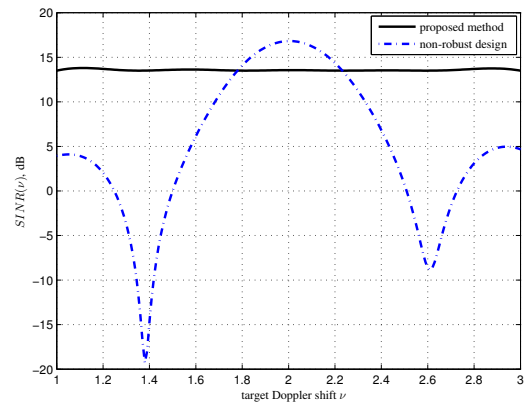

(a)

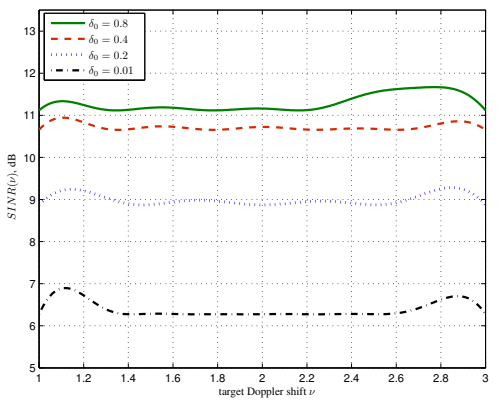

(b)

Fig. 1. (a) comparison of the robust design and non-robust design for a typical example, (b) the effect of the similarity constraint.

$\mathbf{M}$ of the signal-independent interference, it is assumed that $\mathbf{M}_{m, n}=\rho^{|m-n|}$ with parameter $\rho=0.5$. The generalized Barker code is used for sequence $\mathbf{x}_{0}$ [18]. The size of the similarity region is controlled by $\delta_{0}=\delta / e$. The total transmit energy $e$ is supposed to be equal to $N$. The convex optimization problems are solved via the CVX toolbox [19].

We investigate the robustness of the system SINR w.r.t target Doppler shift assuming $\Omega=\left[\nu_{l}, \nu_{u}\right]=[1,3]$ and $\Omega_{c}=$ $\left[\bar{\nu}_{d}-\frac{\epsilon}{2}, \bar{\nu}_{d}+\frac{\epsilon}{2}\right]=[-0.1,0.1]$ [20]; also the result of nonrobust design [8] with given Doppler shift $\nu_{\text {given }}=0.5\left(\nu_{l}+\right.$ $\left.\nu_{u}\right)$ is considered as the benchmark for the comparison. The results are plotted in Fig. 1 (a) for $\delta_{0}=0.5$. It is observed that using the proposed method leads to a robustness for the system performance; furthermore, a significant increase in the minimum value of $\operatorname{SINR}(\nu)$ is observed as compared with that of non-robust design. Note that in this example, the ranks of the optimal $\mathbf{W}_{\star}$ and $\mathbf{X}_{\star}$ were equal to one. Examples of design with various sizes of similarity region are provided in Fig. 1 (b). In this figure, the behavior of the system SINR is shown w.r.t target Doppler shift for $\delta_{0}$ in $\{0.01,0.2,0.4,0.8\}$. The robustness property with respect to the target Doppler shift $\nu$ is observed in all examples. As expected, the larger the $\delta_{0}$, the larger the worst value of the $\operatorname{SINR}(\nu)$. This is due to a larger feasibility set for the optimization problem associated with obtaining $\mathbf{X}$ and the fact that the optimal $\mathbf{W}_{\star}$ and $\mathbf{X}_{\star}$ are rank-one.

Next we consider an example in which the rank of optimal $\mathbf{W}_{\star}$ and $\mathbf{X}_{\star}$ are larger than one. As discussed earlier, the rank of the solutions to the relaxed problem $\mathcal{P}_{1}$ depend on the employed starting point in addition to the design parameters. Note that it was numerically observed that the rank of $\mathbf{X}_{\star}$ is equal to one as long as $\Omega \cap \Omega_{c}=\varnothing$. As to the rank of $\mathbf{W}_{\star}$, a similar observation was made for most of the employed random starting points. Nevertheless, by setting $\Omega=[1,2]$, $\Omega_{c}=[-0.25,0.25], \delta_{0}=0.1$, we find a case for which we have $\operatorname{rank}\left(\mathbf{W}_{\star}\right)=2$ and $\operatorname{rank}\left(\mathbf{X}_{\star}\right)=1$. The SINR associated with the optimal solutions and the employed rank-one decomposition method are illustrated in Fig. 2. For the current example, we have $\nu_{\star}=1.71$ and the best result for the

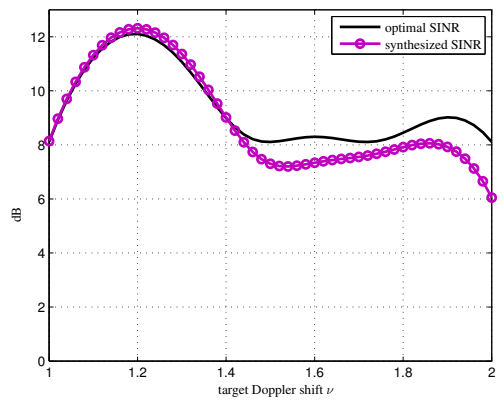

Fig. 2. An example of using the rank-one decomposition method for a case in which $\operatorname{rank}\left(\mathbf{W}_{\star}\right)=2, \operatorname{rank}\left(\mathbf{X}_{\star}\right)=1$.

decomposition method is obtained with $\nu^{\prime}=1.3$ and $\nu^{\prime \prime}=1.5$ (via trying several pairs of $\left(\nu^{\prime}, \nu^{\prime \prime}\right)$ ). It is observed that the synthesized pair of transmit sequence and receive filter follow well the general behavior of the SINR corresponding to the optimal solutions. A minor degradation for the minimum value of the synthesized $\operatorname{SINR}(\nu)$ is also observed due to synthesis loss.

\section{CONCLUDING REMARKS}

A joint max-min design of the transmit sequence and receive filter was considered for cases where the Doppler shift of the target is unknown. A novel method was proposed to tackle the design problem under the similarity constraint. The proposed method consists of a cyclic algorithm (to tackle a relaxed version of the design problem) along with a synthesis stage. We considered a reformulation of $\operatorname{SINR}(\nu)$ by using $\mathbf{W}=\mathbf{w} \mathbf{w}^{H}$ and $\mathbf{X}=\mathbf{x} \mathbf{x}^{H}$, relaxation of the rank-one constraints on the aforementioned matrices, cyclic maximization of the relaxed problem, and a synthesis stage (based on a new rank-one decomposition method). Simulation results showed that employing the proposed method leads to a considerable robustness of the system performance w.r.t the target Doppler shift. 


\section{REFERENCES}

[1] W. D. Rummler, "A technique for improving the clutter performance of coherent pulse trains," IEEE Trans. Aerosp. Electron. Syst., vol. 3, pp. 898-906, Nov. 1967.

[2] D. F. Delong and E. M. Hofstteter, "On the design of optimum radar waveforms for clutter rejection," IEEE Trans. Inf. Theory, vol. 13, pp. 454-463, Jul. 1967.

[3] B. Friedlander, "Waveform design for MIMO radars," IEEE Trans. Aerosp. Electron. Syst., vol. 43, pp. $1227-$ 1238, Jul. 2007.

[4] P. Stoica, J. Li, and Y. Xie, "On probing signal design for MIMO radar," IEEE Trans. Signal Process., vol. 55, no. 8, pp. 4151-4161, 2007.

[5] M. M. Naghsh, M. Modarres-Hashemi, S. ShahbazPanahi, M. Soltanalian, and P. Stoica, "Unified optimization framework for multi-static radar code design using information-theoretic criteria," IEEE Trans. Signal Process., vol. 61, no. 21, pp. 5401-5416, 2013.

[6] S. M. Kay, "Optimal signal design for detection of Gaussian point targets in stationary Gaussian clutter/reverberation," IEEE J. Sel. Topics Signal Process., vol. 1, pp. 31-41, Jun. 2007.

[7] P. Stoica, H. He, and J. Li, "Optimization of the receive filter and transmit sequence for active sensing," IEEE Trans. Signal Process., vol. 60, no. 4, pp. $1730-1740$, Apr. 2012.

[8] A. Aubry, A. De Maio, A. Farina, and M. Wicks, "Knowledge-aided (potentially cognitive) transmit signal and receive filter design in signal-dependent clutter," IEEE Trans. Aerosp. Electron. Syst., vol. 49, pp. 93-117, Jan. 2013.

[9] A. Aubry, A. De Maio, M. Piezzo, A. Farina, and M. Wicks, "Cognitive design of the receive filter and transmitted phase code in reverberating environment," Radar, Sonar Navigation, IET, vol. 6, no. 9, pp. 822833, 2012.

[10] M. M. Naghsh, M. Soltanalian, P. Stoica, and M. Modarres-Hashemi, "Radar code optimization for moving target detection," in EUSIPCO, Marrakech, Morocco, 2013.

[11] A. De Maio, Y. Huang, M. Piezzo, S. Zhang, and A. Farina, "Design of optimized radar codes with a peak to average power ratio constraint," IEEE Trans. Signal Process., vol. 59, pp. 2683-2697, Jun. 2011.
[12] P. Stoica and Y. Selen, "Cyclic minimizers, majorization techniques, and the expectation-maximization algorithm: a refresher,' IEEE Signal Process. Mag., vol. 21, no. 1 , pp. 112 - 114, Jan. 2004.

[13] A. Charnes and W. Cooper, "Programming with linear fractional functionals," Naval Research logistics quarterly, vol. 9, no. 3-4, pp. 181-186, 1962.

[14] T. Roh and L. Vandenberghe, "Discrete transforms, semidefinite programming, and sum-of-squares representation of nonnegative polynomials," SIAM Journal on Optimization, vol. 16, no. 4, pp. 939-964, 2006.

[15] P. Stoica, T. McKelvey, and J. Mari, "MA estimation in polynomial time," IEEE Trans. Signal Process., vol. 48, pp. 1999-2012, 2000.

[16] A. De Maio, S. De Nicola, Y. Huang, S. Zhang, and A. Farina, "Code design to optimize radar detection performance under accuracy and similarity constraints," IEEE Trans. Signal Process., vol. 56, pp. 5618-5629, Nov. 2008.

[17] W. Ai, Y. Huang, and S. Zhang, "New results on Hermitian matrix rank-one decomposition," Mathematical programming, vol. 128, no. 1, pp. 253-283, 2011.

[18] M. Friese, "Polyphase Barker sequences up to length 36," IEEE Trans. Inf. Theory, vol. 42, no. 4, pp. 12481250, 1996.

[19] M. Grant and S. Boyd. (2012, February) CVX package. [Online]. Available: http://www.cvxr.com/cvx

[20] M. Skolnik, Radar Handbook, 3rd ed. New York: McGraw-Hill, 2008. 